\title{
Feasibility study of strain and temperature discrimination in a BOTDA system via Artificial Neural Networks
}

\author{
R. Ruiz-Lombera*a, A. Piccolo ${ }^{\mathrm{b}}$, L. Rodriguez-Cobo ${ }^{\mathrm{a}, \mathrm{c}}$, J.M. Lopez-Higuera ${ }^{\mathrm{a}, \mathrm{c}}, \mathrm{J}$. Mirapeix ${ }^{\mathrm{a}, \mathrm{c}}$ \\ a Photonics Engineering Group, University of Cantabria, 39005, Santander (Spain) \\ bepartment Information Engineering, Università di Padova, 35131 Padova, Italy \\ ${ }^{c}$ CIBER-bbn, Instituto de Salud Carlos III, 28029, Madrid (Spain)
}

\begin{abstract}
Automatic discrimination between strain and temperature in a Brillouin optical time domain analyzer via artificial neural networks is proposed and discussed in this paper. Using a standard monomode optical fiber as the sensing element, the ability of the proposed solution to detect the known changes that the Brillouin gain spectrum exhibits depending on the applied temperature and/or strain will be studied. Experimental results, where different simultaneous strain and temperature situations have been considered, will show the feasibility of this technique.
\end{abstract}

Keywords: BOTDA, stimulated Brillouin scattering, artificial neural network, temperature and strain discrimination

\section{INTRODUCTION}

Distributed sensing via optical fiber sensor systems has become a significant area of research in the last years, given the remarkable features that these solutions offer in terms of monitoring large infrastructures along tens of kilometers with spatial resolutions around the 1-meter limit for standard implementations. While systems based on Raman scattering are limited to temperature measurements ${ }^{1}$, Brillouin scattering enables the possibility of simultaneously estimating both strain and temperature ${ }^{2}$. This inherent advantage exhibited by both spontaneous and stimulated scattering-based solutions may become a drawback given that it is not straightforward to identify the contributions from the above mentioned parameters. In Brillouin-based distributed sensor systems, particularly in Brillouin optical time-domain analyzers (BOTDAs), it is known that the Brillouin frequency shift (BFS) exhibits a linear dependence for both temperature and strain $^{3}$, giving rise to a sensitivity of about $1 \mathrm{MHz} /{ }^{\circ} \mathrm{C}$ and $50 \mathrm{MHz} / 1000 \mu \varepsilon$, respectively. Specialty fibers are commonly employed when only temperature or strain are to be measured, thus trying to avoid a possible contribution from the remaining parameter that might give rise to an erroneous result ${ }^{4}$.

Several authors have proposed different alternatives to perform strain/temperature discrimination in Brillouin-based distributed sensor systems. A simultaneous use of Raman and Brillouin scattering processes in the same system may allow to perform the desired discrimination, as Raman scattering will only provide information on temperature ${ }^{1}$. Other solutions are based on the use of large-effective-area nonzero-dispersion-shifted ${ }^{5}$ (LEAF) or photonic crystal $^{6}$ (PCF) fibers, given that in these cases several Brillouin peaks, with different temperature/strain dependences, appear in the acquired spectra. Polarization maintaining fibers have also been used in this regard by involving several parameters such as the BFS and the associated power and bandwidth ${ }^{7}$.

Apart from the linear dependence between the BFS and the applied strain/temperature change, the Brillouin gain spectrum (BGS) exhibits a different behavior depending on the parameter under analysis: increasing temperatures will provoke narrower BGSs, in addition to a higher gain associated with the BGS; on the contrary, an increasing strain will give rise to wider BGSs and corresponding decreasing powers ${ }^{3}$. These changes are normally too subtle to be taken into account to distinguish between strain and temperature contributions in standard BOTDAs. However, in this paper we propose the employment of artificial neural networks (ANNs) in an attempt to use these features to perform an automatic discrimination of both parameters using a conventional BOTDA implementation.

*ruben.ruiz@unican.es; phone (+34) 942 206736; gif.teisa.unican.es

25th International Conference on Optical Fiber Sensors, edited by Youngjoo Chung, Wei Jin,

Byoungho Lee, John Canning, Kentaro Nakamura, Libo Yuan, Proc. of SPIE Vol. 10323,

103237Z · C 2017 SPIE · CCC code: 0277-786X/17/\$18 · doi: 10.1117/12.2265435 


\section{EXPERIMENTAL ISSUES}

\subsection{Experimental setups}

A complete set of experiments involving simultaneous measurements of strain and temperature was designed to check the performance of the ANN approach. Although some initial measurements were performed in a climatic chamber, the necessity to apply strain to several meters of the fiber under test (FUT) led to the design of the water tank (142 x $32 \mathrm{~cm})$ shown in Fig. 1. A homogeneous water temperature can be achieved via a customized temperature controller, a thermocouple, an electrical resistance and an air pump. On the other hand, a strain setup, formed by two wheels and a positioner, was located inside the tank, allowing to perform a quasi-homogeneous strain to approximately 20 meters of the FUT.

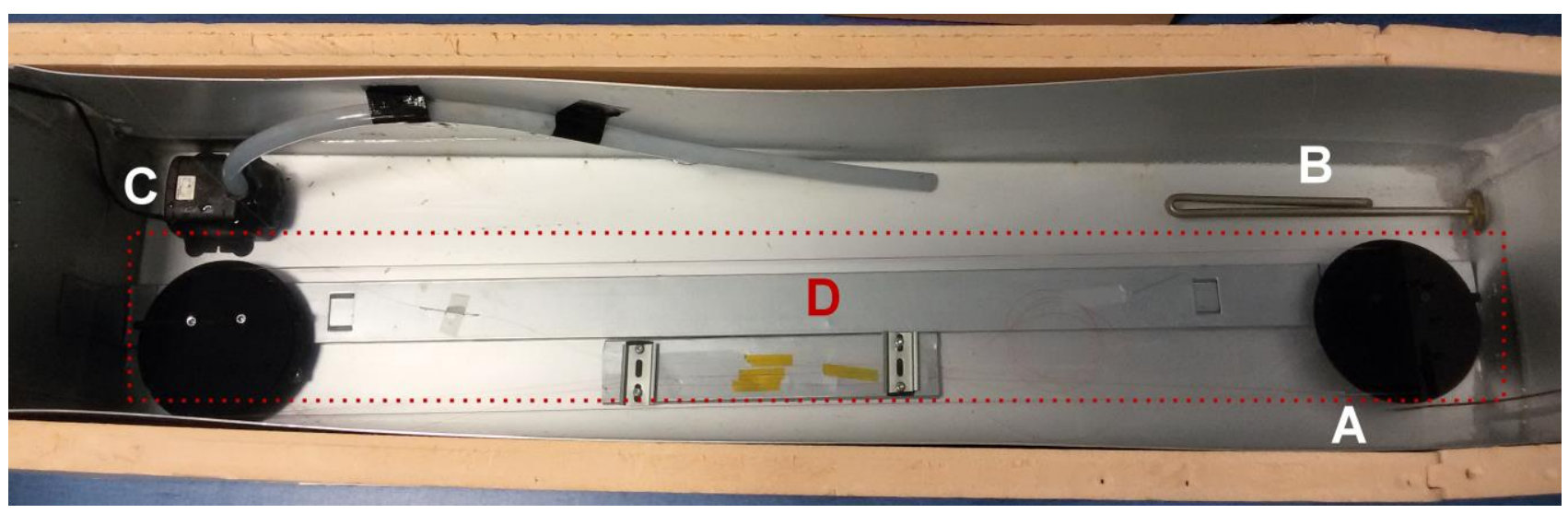

Figure 1. Aluminium tank covered by thermal isolator designed for the strain and temperature experiments: a) thermocouple; b) electrical resistance used to heat the water; c) air pump to get a homogeneus water temperature distribution; d) strain setup formed by two wheels and a positioner (left wheel) to apply strain to the FUT.

A standard BOTDA system, like the one described in ${ }^{8}$, was employed to carry out the distributed measurements. A single laser source emitting at $\lambda=1550.92 \mathrm{~nm}$ generates the light used for both pump and probe waves. The pulsed pump is then created via a semiconductor optical amplifier (SOA), with a pulse width of approximately $12 \mathrm{~ns}$. This wave is amplified via an Erbium-doped fiber amplifier (EDFA) and goes through a polarization scrambler before entering the FUT. The two sidebands of the probe wave are generated with an electro-optical modulator (EOM) and a RF generator. Finally, the detection stage is formed by a fiber Bragg grating allowing to select the lower frequency sideband of the incoming signal and a high-transimpedance gain $125 \mathrm{MHz}$ photodetector and an acquisition card with an averaging of 1024 samples. The FUT comprises a total of $4 \mathrm{~km}$ (2 spools of $2 \mathrm{~km} \mathrm{SMF} \mathrm{-G652B/D),} \mathrm{with} \mathrm{approximately} 20$ meters located inside the water tank, just before the splice between both spools.

\subsection{ANN design}

A simple ANN design was chosen to study the feasibility of the proposed solution: a feedforward network with a supervised backpropagation learning algorithm. The Levenberg-Marquardt optimization was chosen as the training algorithm and the minimum squared error performance function was used to decide whether the training stage is finished. The acquired BOTDA data are divided into training, validation and test stages. The ratio of data used for these stages, the network structure (number of hidden layers and associated number of neurons) and even the way the BOTDA data are fed into the network have been varied to study their influence in the final classification results. The BGSs obtained from the BOTDA traces have been treated in two ways: a) sampling the frequency/intensity values at given percentages in terms of the peak intensity of the BGS $(25,40,50,70,85$ and 95\%) (stripes method) and b) performing a Lorentzian fit and using the associated information. 


\subsection{BOTDA data}

Figure 2 presents an example of the BOTDA data acquired to feed the ANN. The experimental tests were designed considering temperatures from 20 to $72^{\circ} \mathrm{C}$ with steps of $\approx 8^{\circ} \mathrm{C}$. For each temperature, 11 different strain situations were considered, from 0 to 10 turns of the positioning wheel, where 1 turn gives rise to an elongation of $0.5 \mathrm{~mm}$ in the FUT section. Each measurement (strain-temperature pair) was repeated three times. In Fig. 2, the x-axis position denotes the number of samples. As the sampling interval is $0.5 \mathrm{~m}$, the length of the FUT section is $20 \mathrm{~m}$. It can be observed how higher strains provoke an inhomogeneous strain distribution along the FUT, with higher strains associated with the fiber located over the wheels of the strain setup. This is obviously a situation to be avoided, but that can be at the same time useful in evaluating the performance of the ANN to handle noisy data.

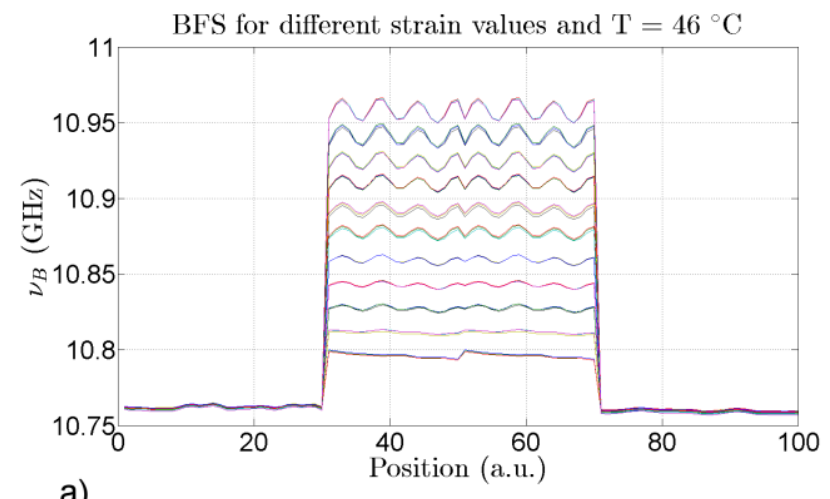

a)

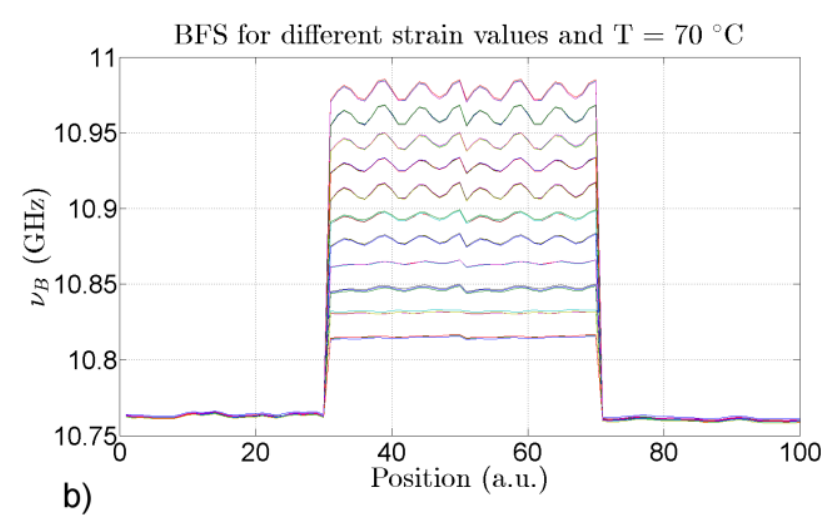

Figure 2. Example of data acquired with the BOTDA system: a) BFSs at the hotspot section for $\mathrm{T}=46^{\circ} \mathrm{C}$ and strains from 0 to 10 turns; a) BFSs at the hotspot section for $\mathrm{T}=70^{\circ} \mathrm{C}$ and strains from 0 to 10 turns.

\section{RESULTS}

Multiple results were obtained modifying the parameters mentioned above, as well as the method to evaluate the ANN output: averaging all the hotspot ANN outputs to obtain a single representative value or counting the ANN outputs and choosing the maximum value of the ANN output vector for strain and temperature. Figure 3 shows an example of the achieved results. Figure 3(a) presents two network configurations where the stripes method has been considered to sample the input data; while Fig. 3(b) shows results associated with the use of the Lorentzian fitting method. In both cases, some problematic tests, those corresponding to 0 strain and 20 and $62^{\circ} \mathrm{C}$, have been eliminated from the dataset in an attempt to improve the classification results. These experiments gave rise to non-consistent results in terms of the remaining data due to different reasons (for example 0 strain was found to be non-homogeneous for different temperatures and $20^{\circ} \mathrm{C}$ was measured at room temperature, thus exhibiting more fluctuations in comparison to other trials).

It can be observed how the best results in these cases are obtained in Fig. 3(b), in particular when using a single hidden layer with 11 and 8 neurons and a ratio of 70/10/20 for training/validation/test. These results are obtained as the average for each ANN configuration, since the simulation was performed twice per each network. The best single results (without averaging) obtained for temperature/strain classification have been of 56.7/86.7\% and 70/73.3\%, using a single hidden layer with 11 neurons a 70/10/20 dataset, the Lorentzian method to choose the input data and average and single maximum output methods, respectively. 

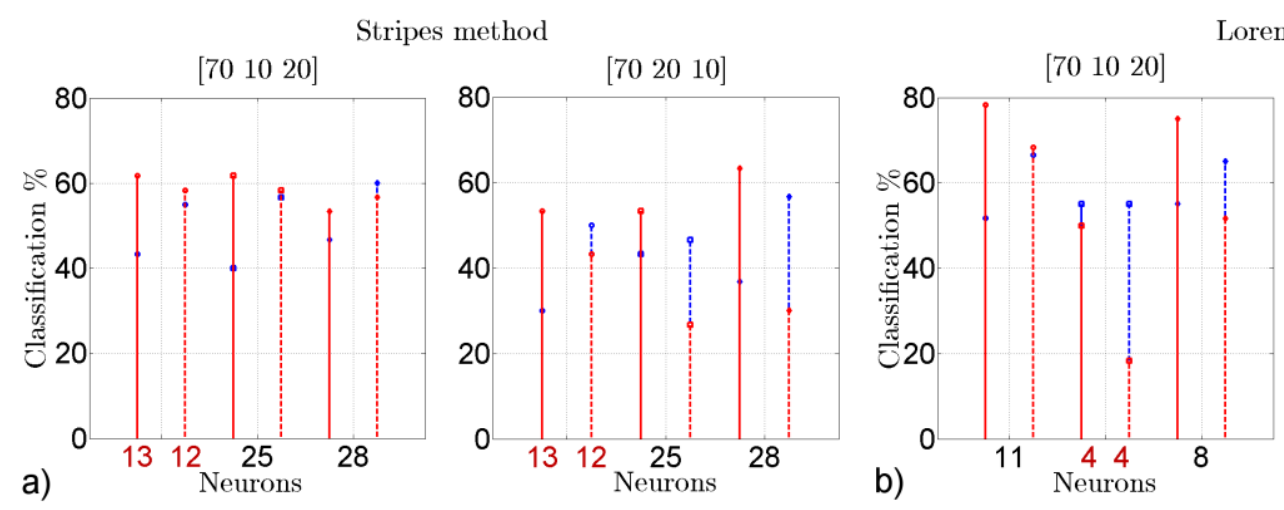

orentzian fit

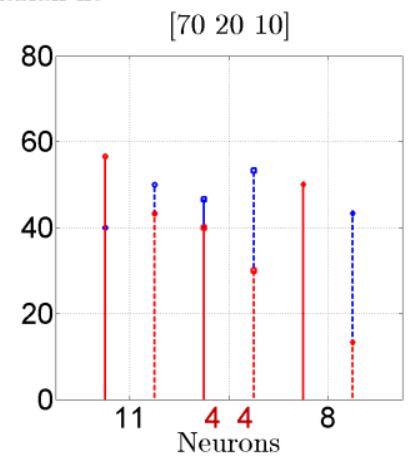

Figure 3. Example of ANN results: a) considering the stripes method to feed the network; b) using the Lorentzian fit method to feed the network. Temperature and strain values are represented in blue and red, respectively. Training/validation/test data ratios are listed above each figure in brackets. Numbers in red in the x-axis indicate the use of two hidden layers (e.g. 1312 indicates the use of two hidden layers with 13 and 12 neurons, respectively); otherwise, a single hidden layer with the indicated number of neurons is considered. Output evaluation: a straight line indicates the use of the average method and a dashed line the single maximum.

\section{CONCLUSIONS}

In this work, we have explored the feasibility of performing temperature/strain discrimination in distributed measurements via a conventional BOTDA system. Different strategies and neuronal network configurations have been investigated, giving rise to classification results that reach the $70-80 \%$ of success. We believe that this is a promising result that suggests that further efforts should be devoted to reach higher classification percentages. On the one hand, experiments should be carefully developed in order to avoid the appearance of inconsistencies in the acquired data. Wider pump pulses should also be considered to avoid a possible widening of the resulting BGS that might make it difficult to detect the different effects generated by temperature and strain changes in the Brillouin spectrum linewidth. Finally, more sophisticated artificial intelligence approaches, such as deep learning techniques, might also be studied.

\section{ACKNOWLEDGEMENTS}

This work has been supported by the projects TEC2013-47264-C2-1-R and TEC2016-76021-C2-2-R.

\section{REFERENCES}

[1] Dakin, J., Pratt, D., Bibby,G., Ross, J., "Distributed optical fibre Raman temperature sensor using a semiconductor light source and detector," Electronics letters 21, 569-70 (1985).

[2] Kurashima, T., Horiguchi, T., Tateda, M., "Distributed-temperature sensing using stimulated Brillouin scattering in optical silica fibers," Optics Letters 15, 1038-40 (1990).

[3] Nikles, M., Thevenaz, L., Robert, P., "Brillouin gain spectrum characterization in single-mode optical fibers," Journal of Lightwave Technology 15, 1842-51 (1997).

[4] Inaudi, D., Glisic, B., "Development of distributed strain and temperature sensing cables," International Society for Optics and Photonics 2005, 222-5 (2005).

[5] Lee, C., Chiang, P., Chi, S., "Utilization of a dispersion-shifted fiber for simultaneous measurement of distributed strain and temperature through Brillouin frequency shift," IEEE Photonics Technology Letters 13 , 1094-6 (2001).

[6] Zou, L., Bao, X., Afshar, S., Chen, L. "Dependence of the Brillouin frequency shift on strain and temperature in a photonic crystal fiber," Optics letters 29, 1485-7 (2004).

[7] Bao, X., Yu, Q., Chen,L., "Simultaneous strain and temperature measurements with polarization-maintaining fibers and their error analysis by use of a distributed Brillouin loss system," Optics letters 29, 1342-4 (2004).

[8] Ruiz-Lombera, R., Mirapeix, J., Laarossi, I., Rodríguez-Cobo, L., Lopez-Higuera, J., "Brillouin frequency shift estimation in BOTDA via subpixel processing," Sixth European Workshop on Optical Fibre Sensors (EWOFS'2016) International Society for Optics and Photonics 2016, 99162L-4 (2016). 